\title{
Structural Sustainability of the Polish Trade System
}

\author{
P. FIEDOR* \\ African Institute of Financial Markets and Risk Management, University of Cape Town, \\ Rondebosch, Cape Town, 7700, South Africa
}

\begin{abstract}
We use multi-region Input-Output databases to show the sustainability of the Polish trade system. Analyses of the robustness of the supply system as a whole are missing in the literature, in strong contrast with a wide variety of network analyses inquiring into the resilience of financial systems. We represent the trade system as a flow network, and use information-theoretic approach to address growth and development of such a system. We perform an analysis of the development, robustness, and structural sustainability of the Polish trade system based on national Input-Output Tables (in current prices) for Poland for the years between 1995 and 2011. As such, we are also able to comment on the changes of the studied characteristics over the years. Further, we compare the results with the results obtained for the global supply system based on the multi-region Input-Output Tables. We find the Polish supply system to be much less organised than the global supply system. We also quantify the effect of the 2008 financial crisis on the size and organisation of the trade system in Poland.
\end{abstract}

DOI: 10.12693/APhysPolA.129.1004

PACS/topics: 89.20.-a, 89.65.Gh

\section{Introduction}

The sustainability of supply chains is of great importance to research and policies [1-5]. Measuring it has not been properly introduced in literature, however. Research on sustainability of the supply chains concentrates on microlevel analysis. A recent study [5] established the need for quantitative measures from all dimensions of sustainability. The framework presented there is still a collection of microlevel measures, however [5].

We introduce a general framework for assessing the structural sustainability of a supply system without understanding all the mechanisms in the microscale, as we find the sustainability inherent in the structure of the system itself. By moving to a macroeconomic perspective, we relax many assumptions and requirements of the methodology, and arrive at interesting results about the system as a whole. The results are more relevant for policy, as it is more feasible to influence the properties of the system, instead of influencing all of its components.

We understand the supply system to be sustainable when it is not only concentrated on growth and development as its only goals, but approaches its development from a systematic viewpoint. That is, we find that sustainability requires a balance between efficiency and resilience [6]. The ecological network analysis (ENA) framework is an appropriate way to analyse sustainability according to this definition.

It is worth noting that international trade was the last strand of economics to move away from sheer theory with very little empirical basis. Two high-quality databases have been constructed in recent years, containing multiregion and national Input-Output (MRIO) tables, which

*corresponding author; e-mail: Pawel.Fiedor@uct.ac.za help in making trade economics truly an empirical science. This paper presents a way to analyse the sustainability of the Polish trade system using network theory and ecological network analysis.

The paper is organised as follows. In Sect. 2 we present methods used to determine the structural sustainability of the Polish trade system, and the MRIO tables describing the Polish trade system. In Sect. 3 we analyse the structure of the Polish trade system between 1995 and 2011 using the presented methodology, and discuss obtained results. In Sect. 4 we conclude the study and propose further research.

\section{Methods}

Goerner et al. [7] have created a framework called quantitative economic development (QED), which can be used to measure healthy development of a complex flow system. This approach to economic sustainability is a precise way to differentiate between healthy development and mere growth [8]. The framework assumes that sustainability of the system is based on the layout and magnitudes of the flows by which energy and information or capital and resources are circulated within it. As the economic structure can be modelled as a map of flows of goods, services, or money circulating across a network [9], such an approach fits naturally for the MRIO tables, which can be easily converted into flow networks.

The growth of a flow network may be easily determined as the sum of all flows, but the inquiry into the sustainability of such system is not trivial. The system efficiency is the capacity of the system to perform its functions in an organised and efficient manner [10], and its resilience is the reserve present in the system, allowing for a diversity of actions to be relied upon in case of shocks or disturbances within the system [11-13]. As both resilience and efficiency are related to the diversity 
and connectivity within the system, we may study them simultaneously [7].

Excessive increases in the system efficiency may make the system more brittle, while increasing diversity and connectivity may make the system more resilient, but in excess renders the system more stagnant. Thus, an optimal mix of the two is desired [6]. We define sustainability of a flow network as the optimal balance of efficiency and resilience, which may be measured by the structural properties of the system. We follow by presenting the underlying mathematics. Goerner et al. [7] show that for natural systems optimal sustainability is situated closer to resilience than efficiency. It is unclear whether this is also true for economic systems, however.

We calculate the growth of a system as total system throughput (TST), the sum of all flows in the studied system. It reflects the size and overall activity of the system, but cannot be used for more thorough analyses of the economic properties of the system. We define $T_{i j}$ as the flow of capital from sector $i$ to sector $j$. Further, we define the aggregate as:

$$
T_{i .}=\sum_{j} T_{i j}
$$

and understand that it represents the rate at which capital leaves sector $i$ at a given time (a year). Conversely, the measure

$$
T_{. j}=\sum_{i} T_{i j}
$$

represents the rate at which capital enters sector $j$ at a given time. Then we may define:

$$
\mathrm{TST}=T_{. .}=\sum_{i, j} T_{i j}
$$

as the total activity of the system (TST). We understand growth of the system as an increase in TST.

We understand development as an increase in the organisation of the system, independently of its size. The more organised a system, the more information it can provide [14]. The average mutual information (AMI) measures how the flows in a system constrain each other, and estimates such organisation, so that an increase in AMI measures the development of the system. For the theoretical properties and derivation of average mutual information see Refs. [14, 15], here we only define it for the above-mentioned flow networks, as:

$$
\mathrm{AMI}=k \sum_{i, j} \frac{T_{i j}}{T_{. .}} \log \left(\frac{T_{i j} T_{. .}}{T_{. j} T_{i .}}\right) .
$$

$k$ is of secondary importance, and defines the unit of information. We use bits in this study. AMI is always positive, and its maximum is constrained by the Shannon entropy [15], or the average indeterminacy for the whole system

$$
H=-k \sum_{i, j} \frac{T_{i j}}{T_{. .}} \log \left(\frac{T_{i j}}{T_{. .}}\right) .
$$

The AMI measures the regular, orderly, coherent and efficient behaviours within the system [14]. Higher AMI values reflect stronger constraints on the movement of flows within the system. In such systems, certain links tend to be more efficient in transferring flows, and consequently become increasingly important within the system. Importantly, this may lead to lack of resilience if only few links within the flow network encompass a large part of the system operations.

The rest of the model allows for the precise analysis of the trade-off mentioned above. The product of TST and AMI is defined as ascendency [6]. Ascendency combines the activity and organisation of the system, and provides a single measure of the process of growth and development of the system

$$
A=\mathrm{TST} \times \mathrm{AMI}=\sum_{i j} T_{i j} \log \left(\frac{T_{i j} T_{. .}}{T_{. j} T_{i .}}\right) .
$$

Scaling $H$ by TST defines the development capacity of the studied system, which may be understood as the upper bound on the ascendency of the system

$$
C=\mathrm{TST} \times H=-\sum_{i j} T_{i j} \log \left(\frac{T_{i j}}{T_{. .}}\right) .
$$

The difference between development capacity and ascendency is called the resilience of the system, and can be thought of as an opposite to ascendency

$$
R=C-A \text {. }
$$

We use the World Input-Output database, founded by the European Commission (WIOD World Input-Output Database), for this purpose. In particular, we use National Input-Output Tables for Poland, released November 2013. These tables provide World Input-Output Tables in current prices, denoted in millions of dollars. The database covers 35 sectors of the Polish economy for the period from 1995 to 2011. The list of these sectors can be found on the above-mentioned website. Currency networks are build from nodes (these represent sectors) and flows between them (flows of goods, represented in monetary terms). We do not explicitly model the outside of the system, concentrating on the internal structure of the studied economy.

\section{Results and discussion}

We start with the analysis of the growth of the system. Input-Output Tables between 1995 and 2011 were converted into flow networks and values of TST have been calculated according to Eq. (3). Figure 1 presents the growth of TST over the studied years. We observe that for most of this period the total throughput of the system grew exponentially (if we fit an exponential regression we get $R^{2}=0.91$ ). We may also gauge the loss in this growth due to the recent financial crisis, which we may call trade-at-risk, to misquote the recently popular GDP-at-risk.

We also analyse the AMI, which describes the organisation and development of the system. The resulting values of AMI for the Polish supply system for the studied years are presented in Fig. 2. We observe that average mutual 


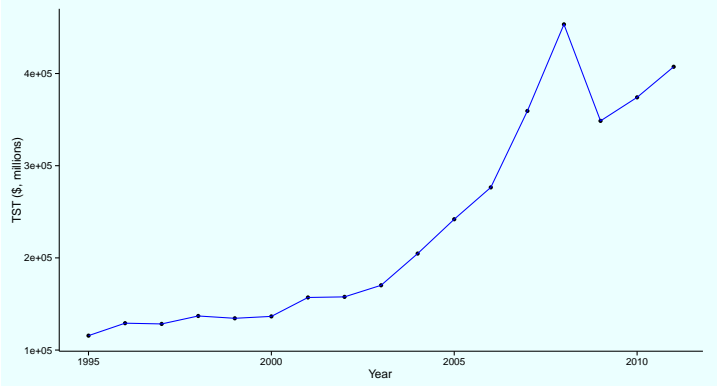

Fig. 1. Total throughput (TST) of the Polish trade system over the studied years. We observe an exponential growth of the international trade system over the years.

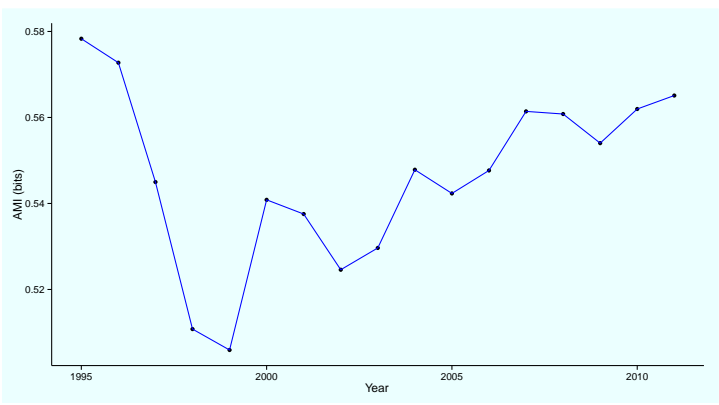

Fig. 2. Average mutual information of the Polish trade system over the studied years. We observe a stable development of the system over the years.

information fluctuated between 0.5 and 0.58 bits. This is comparable with around 0.5 bits for Beijing economy [14], but smaller than the global supply system, which oscillates around 2.75 bits. It appears that the Polish supply system is relatively well organised, but not on par with the global supply system.

Ascendency and development capacity have been calculated for the studied system, and are presented in Fig. 3. We observe that both measures grow exponen-

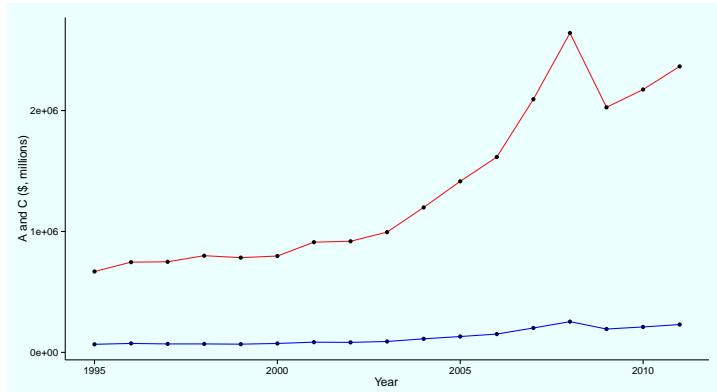

Fig. 3. Ascendency (A, blue line below) and development capacity $(\mathrm{C}$, red line above) of the Polish trade system over the studied years. We observe a stable development of the system over the years.

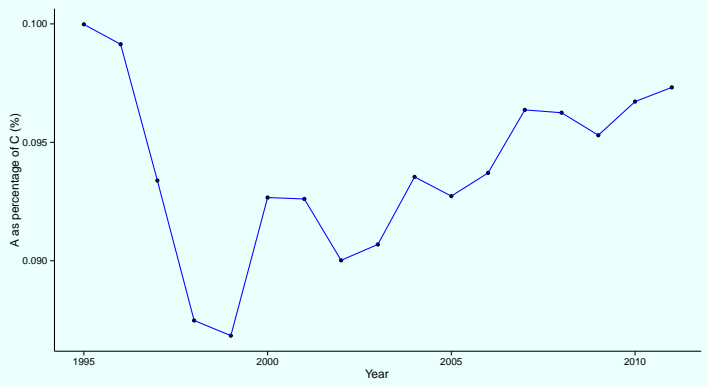

Fig. 4. Ascendency as percentage of development capacity of the Polish trade system over the studied years. We observe very small changes over the studied period, perhaps hinting that the Polish supply system is stable and mature, but the ascendency contains a small percentage of the system capacity.

TABLE I

Contribution of TST and AMI to ascendency of the Polish trade system. The ascendency of the system is largely driven by its total throughput.

\begin{tabular}{c|c|c|c|c}
\hline \hline & 1996 & 1997 & 1998 & 1999 \\
\hline AMI & $-9.4 \%$ & $88.9 \%$ & $-14.2 \%$ & $34.1 \%$ \\
TST & $109.6 \%$ & $11.1 \%$ & $114.2 \%$ & $65.9 \%$ \\
\hline & 2000 & 2001 & 2002 & 2003 \\
\hline AMI & $81.4 \%$ & $-4.6 \%$ & $120.0 \%$ & $11.1 \%$ \\
TST & $18.6 \%$ & $104.6 \%$ & $-20.0 \%$ & $88.9 \%$ \\
\hline & 2004 & 2005 & 2006 & 2007 \\
\hline AMI & $15.5 \%$ & $-6.5 \%$ & $6.8 \%$ & $8.7 \%$ \\
TST & $84.5 \%$ & $106.5 \%$ & $93.2 \%$ & $91.3 \%$ \\
\hline & 2008 & 2009 & 2010 & 2011 \\
\hline AMI & $-0.5 \%$ & $4.4 \%$ & $16.8 \%$ & $6.1 \%$ \\
TST & $100.5 \%$ & $95.6 \%$ & $83.2 \%$ & $93.9 \%$
\end{tabular}

tially, at roughly the same pace, which means that the development of the system is not in danger of being blocked any time soon.

We may observe this in Fig. 4, which shows ascendency as percentage of development capacity. We see very little variation, hinting that the system grows in a healthy and sustainable way. The ascendency contains only about $10 \%$ of the system capacity, however. Comparing this with about $30 \%$ for the global supply system, we see that the Polish supply system is not very efficient. Policy makers may want to find ways to strengthen the efficiency of this system. On the other hand, the system appears very resilient, which may have played a role in the unveiling of the crisis of 2007-2008, when Poland did not end up in recession.

Finally, since ascendency is a compound variable, based on TST and AMI, we may decompose the changes in the ascendency in parts which were contributed by both of its constituents (see Ref. [14] for details). We present these results for each two year periods (denoted as the last of two years) in Table I. We see that the results vary across the studied period, but the growth of ascendency appears to be mostly based on the growth in the 
throughput of the system, rather than its organisation. This may be due to the system being already mature. But it also hints at the possibility of stagnation.

\section{Conclusions}

We have found that the Polish supply system grew exponentially, similarly with respect to the organisation of the system and its development capacity. While the crisis of 2007-2008 has shifted this growth downwards, the system appears to be developing in a sustainable manner, although it is mostly based on the growth in the throughput of the system, rather than its organisation, which may suggest some degree of stagnation. The ascendency constitutes $10 \%$ of the system capacity, compared with about three times as much for the global supply system. Policy makers should try to make policies to either strengthen the flows with positive contributions to ascendency, or weaken the flows which have negative effect, starting with the ones with the biggest magnitudes, as these would give the highest effect. Further studies should look into analysing other supply system based on other databases, and to answer specific policy questions, perhaps analysing specific contributions of sectors and countries to the development and sustainability of the global trade system.

\section{References}

[1] G. Barbiroli, Technovation 16, 341 (1996).

[2] J. Sarkis, Greener Manufacturing and Operations: From Design to Delivery and Back, Greenleaf, Sheffield 2001, p. 150.
[3] K.-H. Robert, B. Schmidt-Bleek, J. Aloisi de Larderel, G. Basile, J.L. Jansen, R. Kuehr, P. Price Thomas, M. Suzuki, P. Hawken, M. Wackernagel, J. Clean. Prod. 10, 197 (2002).

[4] S.A. Waage, K. Geiser, F. Irwin, A.B. Weissman, M.D. Bertolucci, P. Fisk, G. Basile, S. Cowan, H. Cauley, A. McPherson, J. Clean. Prod. 13, 1145 (2002).

[5] T.W. Sloan, J. Global Bus. Manag. 6, 92 (2010).

[6] R.E. Ulanowicz, S.J. Goerner, B. Lietaer, R. Gomez, Ecological Complexity 6, 27 (2009).

[7] S.J. Goerner, B. Lietaer, R.E. Ulanowicz, Ecol. Econ. 69, 76 (2009).

[8] H.E. Daly, Beyond Growth: The Economics of Sustainable Development, Beacon Press, Boston 1997.

[9] W. Leontief, The Structure of the American Economy, 1919-1939, Oxford University Press, New York 1951.

[10] R.M. May, Nature 238, 413 (1972).

[11] C.S. Holling, Annu. Rev. Ecol. Syst. 4, 1 (1973).

[12] W.C. Clark, R.E. Munn, Sustainable Development of the Biosphere, Cambridge University Press, Cambridge 1986, p. 291.

[13] B.H. Walker, J.M. Anderies, A.P. Kinzig, P. Ryan, Exploring Resilience in Social-Ecological Systems: Comparative Studies and Theory Development, CSIRO Publ., Collingwood, Victoria 2006.

[14] J. Huang, R.E. Ulanowicz, PLoS ONE 9, e100923 (2014).

[15] T. Cover, J. Thomas, Elements of Information Theory, Wiley, New York 1991. 